\title{
Application of the flash method to rods and tubes
}

\author{
Agustín Salazar ${ }^{1}$, Alberto Oleaga $^{1}$, Fernando Alonso $^{2}$ and Idurre Sáez-Ocáriz ${ }^{2}$ \\ ${ }^{1}$ Departamento de Física Aplicada I, Escuela Técnica Superior de Ingeniería, Universidad del País \\ Vasco, Alameda Urquijo s/n, 48013 Bilbao,Spain.E-Mail: agustin.salazar@ehu.es \\ ${ }^{2}$ Centro de Tecnologías Aeronáuticas (CTA), Parque Tecnológico de Álava, Juan de la Cierva 1, \\ 01510 Miñano, Spain.
}

The flash method is the most acknowledge technique to measure the thermal diffusivity at high temperatures. It consists of heating the front surface of an opaque slab by a short laser pulse and detecting the temperature evolution at its rear surface. The thermal diffusivity is obtained by measuring the time corresponding to the half maximum of the temperature rise $\left(t_{1 / 2}\right)$, that is related to the thermal diffusivity through the expression: $t_{1 / 2}=0.1388 L^{2} / D$, where $L$ is the sample thickness and $D$ is the thermal diffusivity. This procedure works under ideal conditions: negligible laser pulse duration and heat losses.

We have extended the classical flash method to be used with rods and tubes. We proceed as follows. First, the temperature distribution when these samples are illuminated by a modulated light beam is calculated. Then, using the inverse Laplace transform, the temperature evolution of the sample after being heated by a short light pulse is obtained.

Let us consider an infinite and opaque hollow cylinder with an outer radius $a$ and an inner radius $b$, that is illuminated uniformly by a modulated light beam of intensity $I_{o}$ and frequency $f(\omega$ $=2 \pi f)$. Its cross-section is shown in Fig. 1 . The temperature oscillation at any point of the cylinder can be written as ${ }^{1,2}$

$$
T(r, \phi, \omega)=\sum_{m=-\infty}^{\infty} A_{m} J_{m}(q r) e^{i m \phi}+\sum_{m=-\infty}^{\infty} B_{m} H_{m}(q r) e^{i m \phi}
$$

where $q=\sqrt{i \omega / D}$ is the thermal wave vector, and $J_{m}$ and $H_{m}$ are the $m$ th order of the Bessel and Hankel functions of the first kind respectively. The first term in Eq. (1) represents the ingoing cylindrical thermal wave starting at the sample surface, while the second one is the corresponding reflected wave at the inner surface. $A_{m}$ and $B_{m}$ are obtained from the heat flux continuity at the cylinder

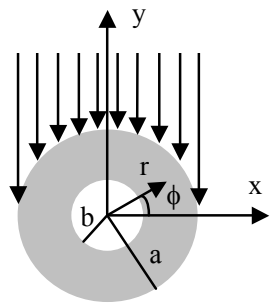

Fig.1.- Geometry surfaces. In this way an analytical solution is obtained

$$
T(r, \phi, \omega)=\frac{I_{o}}{2 K q} \sum_{m=-\infty}^{\infty} \frac{(-i)^{m}}{\pi\left(1-m^{2}\right)} \cos \left(m \frac{\pi}{2}\right) e^{i m \phi} \times \frac{H_{m}^{\prime}(q b) J_{m}(q r)-J_{m}^{\prime}(q b) H_{m}(q r)}{J_{m}^{\prime}(q a) H^{\prime}{ }_{m}(q b)-J^{\prime}{ }_{m}(q b) H^{\prime}{ }_{m}(q a)}
$$

where $J^{\prime}{ }_{m}$ and $H^{\prime}{ }_{m}$ are the derivatives of the Bessel and Hankel functions respectively. A simplified expression can be found for a solid cylinder by making $b=0$.

Now, using the inverse Laplace transform, the temperature evolution after the absorption of a light pulse can be calculated. Following this procedure the temperature rise of the rear surface of three stainless steel samples $\left(K=14.5 \mathrm{Wm}^{-1} \mathrm{~K}^{-1}, D=3.8 \mathrm{~mm}^{2} \mathrm{~s}^{-1}\right)$ after the absorption of a Dirac pulse has been simulated: (a) a $3 \mathrm{~mm}$-thick-slab, (b) a solid cylinder of $3 \mathrm{~mm}$ in diameter whose temperature is measured at the bottom pole, $\phi=-\pi / 2$, and (c) a hollow cylinder with an outer diameter of $3 \mathrm{~mm}$ and an inner diameter of $2 \mathrm{~mm}$, whose temperature is measured at $\phi=-\pi / 2$. Their normalized temperature histories are shown in Fig. 2. Calculations performed for a wide variety of materials indicate that the time required by the back surface to reach the half of the maximum 
temperature rise $\left(t_{1 / 2}\right)$ only depends on the thermal diffusivity and on the sample size, through the equation:

$$
t_{1 / 2}=A \frac{d^{2}}{D}
$$

where $d$ is the thickness in the case of a slab or the diameter $(2 a)$ in the case of a cylinder. For slabs $A$ is the well-known 0.1388 , while for solid cylinders we found $A=0.1068$. A simple formula has not been encountered for hollow cylinders.

The validity of the theory has been tested experimentally by measuring the following AISI-304 stainless steel samples: A rod whose diameter is $4 \mathrm{~mm}$, a

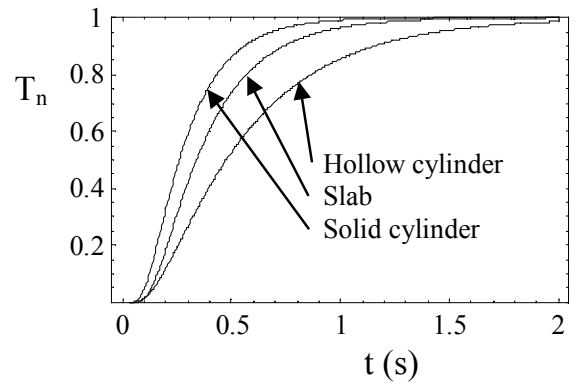

Fig.2.- Calculation of normalized temperature hollow cylinder with an outer diameter of $2.05 \mathrm{~mm}$ and an inner diameter of $1.55 \mathrm{~mm}$, and a $2 \mathrm{~mm}-$ thick-plate that has been used as a reference. The samples have been illuminated by a $6 \mathrm{~kJ}$ flash lamp and their rear surface temperature has been measured by an infrared camera (Thermacam SC 2000 from FLIR Systems) at a frequency rate of 50 frames per second.

In Fig. 3 the temperature rise after the flash light for the three samples under study is shown by dots. In the case of the two cylindrical samples the temperature is the average of 100 pixel placed along the cylinder axis. In the three cases the temperature reaches a constant value at long times after the flash light, indicating that the influence of heat losses is negligible. Using Eq. (3), $\mathrm{D}=3.76 \pm 0.10 \mathrm{~mm}^{2} / \mathrm{s}$ and $\mathrm{D}$ $=3.70 \pm 0.14 \mathrm{~mm}^{2} / \mathrm{s}$ are obtained for the slab and the rod respectively. The continuous lines in Fig. 3 are the fit to the theoretical model. The fitted thermal diffusivity of the tube is $3.84 \pm 0.16 \mathrm{~mm}^{2} / \mathrm{s}$, while for the slab and for

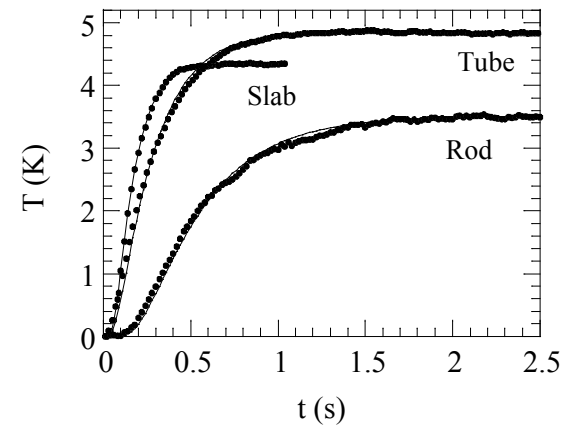

Fig. 3.- Experimental results the rod the same thermal diffusivity values as those found using Eq. (3) are obtained. All values are consistent and fall inside the typical thermal diffusivity values of AISI-304 that can be found in the literature $\left(3.7-4.0 \mathrm{~mm}^{2} / \mathrm{s}\right)$.

Finally, let us compare the flash method as applied to a slab and to the inner side of a tube (i.e., the temperature is measured at $r=b$ and $\phi=+\pi / 2$ ) with the same thickness as that of the slab. It is surprising that the corresponding temperature rise curves are almost indistinguishable even for tubes of small radii. Figure 4 shows the error in the calculated thermal diffusivity of an AISI-304 tube $2 \mathrm{~mm}$ thick as a function of the outer diameter, $a$, when Eq. (3) with $\mathrm{A}=0.1388$ is applied. This result suggests the possibility of applying the classical flash method to a wide variety of curved

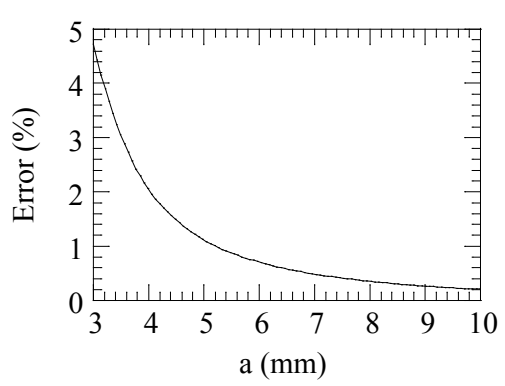

Fig. 4.- Thermal diffusivity error plates.

This work has been supported by the Ministerio de Educación y Ciencia (MAT2005-02999).

${ }^{1}$ J. Sinai and R.C. Waag, J. Acous. Soc. Am. 83, 1729 (1988).

${ }^{2}$ Y.S. Joo, J.G. Ih, and M.S. Choi, J. Acous. Soc. Am. 103, 900 (1998). 